\title{
Incomplete Variable Multigranulation Rough Sets Decision
}

\author{
Ming Zhang ${ }^{1,2, *}$, Weiyan $\mathrm{Xu}^{3}$, Xibei Yang ${ }^{1}$ and Zhenmin Tang ${ }^{2}$ \\ ${ }^{1}$ School of Computer Science and Engineering, Jiangsu University of Science and Technology, Zhenjiang Jiangsu, 212003, China \\ ${ }^{2}$ School of computer science and technology, Nanjing University of science and technology, Nanjing Jiangsu, 210094, China \\ ${ }^{3}$ School of Mathematics and Physics, Jiangsu University of Science and Technology, Zhenjiang Jiangsu, 212003, China
}

Received: 29 May. 2013, Revised: 3 Oct. 2013, Accepted: 4 Oct. 2013

Published online: 1 May. 2014

\begin{abstract}
Recently, a multigranulation rough set (MGRS) has become a new direction in rough set theory, which is different from the Pawlake's rough set since the former takes multiple granulations on the universe into account. In this paper, by analyzing the limitations of optimistic multigranulation rough set (OMGRS) and pessimistic multigranulation rough set (PMGRS) in incomplete information system, the incomplete variable multigranulation rough set (VMGRS) is proposed, and the relationships among VMGRS, OMGRS and PMGRS are deeply explored, From the properties, it can be found that OMGRS and PMGRS are the special cases compared to our VMGRS. Furthermore, several important measurements are introduced into the VMGRS; it is shown that the measurements of the VMGRS are between the measurements of OMGRS and PMGRS. Finally, some numerical examples are employed to substantiate the conceptual arguments.
\end{abstract}

Keywords: OMGRS, PMGRS, VMGRS, Measurement, Incomplete information system

\section{Introduction}

Rough set [1,2], proposed by Pawlak, is a powerful tool, which can be used to deal with the inconsistency problems by separation of certain and doubtful knowledge extracted from the exemplary decisions. Though Pawlak's rough set theory has been demonstrated to be useful in the fields such as knowledge discover [3, 4], decision analysis [5,6], data mining [7,8], pattern recognition [9], artificial intelligence [10], medical diagnosis [11] and so on.

It is well known that lower and upper approximation operators in Pawlak's rough set are defined by an equivalence relation (indiscernibility relation). However, due to the existence of uncertainty and complexity of particular problems, the equivalence relation is too restrictive in many practical applications. To overcome this limitation, Pawlak's rough sets has been extended to several interesting and meaningful general models by proposing other binary relations in recent years, which include similarity relation based rough set [12], tolerance relation based rough set $[9,13,14,15]$, dominance relation based rough set [16], covering based rough set $[17,18]$, fuzzy rough set $[19,20,21,22]$ and others [23].
From the viewpoint of the granular computing, an equivalence relation on the universe can be regarded as a granulation, and a partition (induced by the equivalence relation) on the universe can be regarded as a granulation space, a equivalence class can be regarded as a knowledge granule $[24,25,26]$. So, the above expanded rough set models are based on single granulation, they also called the single granulation rough sets. However, it should be noticed that in $[25,27,28]$, Qian et al. argued that we often need to describe concurrently a target concept through multiple granulations on the universe according to a user's requirements or targets of problem solving. Therefore, they proposed the concept of Multigranulation Rough Set (MGRS) model. In fact, the basic idea of MGRS has been also discussed by Khan et al. in [7]. Following such work, the MGRS are extended in incomplete based MGRS [25], dominance based MGRS [26], neighborhood based MGRS [29], Fuzzy MGRS $[31,30]$, and so on $[32,33,34]$.

The MGRS model can be classified into two parts: one is the optimistic multigranulation rough set (OMGRS) and the other is pessimistic multigranulation rough set (PMGRS). By analyzing OMGRS and PMGRS,

\footnotetext{
*Corresponding author e-mail: zm_fred@163.com
} 
we can see that OMGRS decision is too relax since if only one granulation satisfies with the inclusion condition between the knowledge granule and the target concept, then the object should belong to the lower approximation. On the other hand, PMGRS decision is too strict since if all of the granulations satisfy with the inclusion condition between the knowledge granule and the target, then the object may belongs to the lower approximation.

The purpose of this paper is study MGRS in incomplete decision information system and propose a new multigranulation rough set, which is referred to as the variable multigranulation rough set (VMGRS), In our VMGRS decision, threshold $\beta$, is used to control the number of granulations, which satisfy with the inclusion condition between the knowledge granule and the target concept. Only when the number of granulations reaches to a certain amount, the object may be belongs to the lower approximation. Such number can be controlled by a threshold $\beta$. Through the adjustment of the threshold $\beta$, the limitations of OMGRS too relax and PMGRS too strict can be overcome.

The paper is organized as following. In Section 2, the rough set and MGRS are briefly introduced. In Section 3, the VMGRS models are proposed, the immediate properties about VMGRS are also addressed. In Section 4, several important measurements are introduced into our VMGRS and then the relationships among measurements of OMGRS, PMGRS and VMGRS are discussed. In Section 5, Experiments on UCI data sets show that VMGRS is a generalization of both OMGRS and PMGRS. Results are summarized in Section 6.

\section{Preliminary knowledge on rough sets}

\subsection{Single-granulation rough set}

Formally, an information system(IS) can be considered as a 4-tuple $I S=<U, A T, V, f>$, where $U$ is a nonempty finite set of objects (called the universe), $A T$ is a nonempty finite set of attributes, $V$ is regard as the domain of all attributes and $V=V_{A T}=\bigcup_{a \subset A T} V_{a}, \forall x \in U$, $f(a, x)$ is the value the $x$ hold on $a(a \in A T)$.

In particular, if $A T=C \cup D$ and $C \cap D=\emptyset$, where the $C$ is called condition attribute sets and the $D$ is called decision attribute sets, then $\langle U, C \cup D, V, f\rangle$ is also regard as a decision information system(DIS).

If there exists $x \in U$ and $a \in A T$, such that $f(x, a)$ is unknown, the unknown value is denoted by " $*$ " in this paper, then the $<U, C \cup D, V, f>$ is also regard as an incomplete decision information system(IDIS). We assume here that at least one of the states of $x$ in terms of $A$ is certain where $A \subseteq A T$, i.e. $a \in A$ such that $f(x, a)$ is known. Thus, $V=V_{C} \cup V_{D} \cup\{*\}$.
Definition 1.Suppose that $\langle U, C \cup D, V, f>$ is an IDIS, $\forall A \subseteq C$, a binary relation $\operatorname{SIM}(A)$ can be defined as [13]

$$
\begin{aligned}
\operatorname{SIM}(A)=\left\{(x, y) \in U^{2}:\right. & f(x, a)=f(y, a) \vee f(x, a)=* \\
& \vee f(y, a)=*, \forall a \in A\} .
\end{aligned}
$$

The binary relation $\operatorname{SIM}(A)$ is a tolerance relation since it is reflexive and symmetric. From the relation $\operatorname{SIM}(A)$, we can introduce a cover on the universe $U$, this cover can be denoted by $U / \operatorname{SIM}(A) . \forall x \in U$, We denote the tolerance class including $x$ by $\operatorname{SIM}_{A}(x)$, such $\operatorname{SIM}_{A}(x)=\{y \in U:(x, y) \in \operatorname{SIM}(A)\}$.

From the viewpoint of the granular computing, the relation $\operatorname{SIM}(A)$ can be regard as a granulation, the cover $U / \operatorname{SIM}(A)$ is referred to as a granulation space, each tolerance class $\operatorname{SIM}_{A}(x)$ may be viewed as a knowledge granule consisting of indistinguishable elements.

By tolerance relation $\operatorname{SIM}(A), \forall X \subseteq U$, we can derive the lower and upper approximations of an arbitrary subset $X$ of $U$, They are defined as

$\underline{A}(X)=\left\{x \in U: \operatorname{SIM}_{A}(x) \subseteq X\right\}$.

$\bar{A}(X)=\left\{x \in U: \operatorname{SIM}_{A}(x) \cap X \neq \emptyset\right\}$.

The pair $[\underline{A}(X), \bar{A}(X)]$ is referred to as rough set of $X$ with respect to the set of attributes $A$. Obviously, this rough set is constructed on the basis of one and only one granulation, it is then regarded as the single-granulation rough set model.

\subsection{Multigranulation rough sets}

The MGRS is different from single granulation rough set since the former is constructed on the basis of the multiple granulations instead of a single one.

\subsubsection{Optimistic multigranulation rough set}

In the optimistic multigranulation rough set(OMGRS), the target is approximated through the multiple granulations. In lower approximation, the word optimistic is used to express the idea that in multiple independent granulations, we need only at least one of the granulation to satisfy with the inclusion condition between knowledge granule and target concept. The upper approximation of OMGRS is defined by the complement of the lower approximation.

Definition 2.Let $I=<U, C \cup D, V, f>$ be an IDIS in which $A_{1}, A_{2}, \cdots, A_{m} \subseteq C$, then $\forall X \subseteq U$, the OMGRS lower and upper approximations are denoted by $\underline{\sum_{i=1}^{m} A_{i}}{ }^{O}(X)$ and ${\overline{\sum_{i=1}^{m} A_{i}}}^{O}(X)$, respectively [27],

$\sum_{i=1}^{m} A_{i}^{O}(X)=\left\{x \in U: \operatorname{SIM}_{A_{1}}(x) \subseteq X \vee \operatorname{SIM}_{A_{2}}(x) \subseteq X \vee \cdots\right.$

$\left.\operatorname{VSIM}_{A_{m}}(x) \subseteq X\right\}$.
$\bar{\sum}_{i=1}^{m} A_{i}(X)=\sim \sum_{i=1}^{m} A_{i}(\sim X)$. 
where $\operatorname{SIM}_{A_{i}}(x)(1 \leq i \leq m)$ is a knowledge granule of $x$ in terms of the granulation $\operatorname{SIM}(A), \sim X$ is the complement of set $X$.

By the lower and upper approximations $\underline{\sum_{i=1}^{m} A_{i}{ }^{O}(X)}$ and $\overline{\sum_{i=1}^{m} A_{i}}{ }^{O}(X)$, the OMGRS boundary region of $X$ is

$$
B N_{\sum_{i=1}^{m} A_{i}}(X)=\overline{\sum_{i=1}^{m} A_{i}}(X)-\sum_{i=1}^{m} A_{i}^{O}(X) .
$$

\subsubsection{Pessimistic multigranulation rough set}

In pessimistic multigranulation rough set (PMGRS) [28], the target is still approximated through the multiple granulations. However, it is different from the optimistic case. In lower approximation, the word pessimistic is used to express the idea that we need all of the granulations to satisfy with the inclusion condition between knowledge granule and target concept. The upper approximation of PMGRS is also defined by the complement of the lower approximation of PMGRS.

Definition 3.Let $I=<U, C \cup D, V, f>$ be an IDIS in which $A_{1}, A_{2}, \cdots, A_{m} \subseteq C$, then $\forall X \subseteq U$, the PMGRS lower and upper approximations are denoted by $\underline{\sum_{i=1}^{m} A_{i}^{P}}(X)$ and ${\overline{\sum_{i=1}^{m} A_{i}}}^{P}(X)$, respectively,

$$
\begin{aligned}
\sum_{i=1}^{m} A_{i}(X)= & \left\{x \in U: \operatorname{SIM}_{A_{1}}(x) \subseteq X \wedge \operatorname{SIM}_{A_{1}}(x) \subseteq X \wedge \cdots\right. \\
& \left.\wedge \operatorname{SIM}_{A_{m}}(x) \subseteq X\right\} ; \\
\bar{\sum}_{i=1}^{m} A_{i}(X)= & \sim \sum_{i=1}^{m} A_{i}^{P}(\sim X) .
\end{aligned}
$$

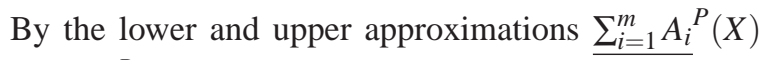
and ${\overline{\sum_{i=1}^{m} A_{i}}}^{P}(X)$, the PMGRS boundary region of $X$ is

$$
B N_{\sum_{i=1}^{m} A_{i}}^{P}(X)=\overline{\sum_{i=1}^{m} A_{i}}(X)-\sum_{i=1}^{m} A_{i}^{P}(X) .
$$

\section{3 variable multigranulation rough set}

By Definition 2, we can see that the OMGRS is too relax since if only one tolerance class(knowledge granule) satisfies with the set containment to the target concept, then the object should belong to the lower approximation. On the other hand, by Definition 3, we can see that the PMGRS is too strict since if all of the tolerance classes satisfy with the set containment to the target concept, then the object belongs to the lower approximation.

To solve such problem, we will propose a new multigranulation rough set approach, which is referred to as the incomplete variable multigranulation rough
set(VMGRS). In our incomplete VMGRS approach, a threshold $\beta$ will be used to control the number of tolerance classes, which satisfy with the set containment to the target concept.

Definition 4.Let $I=<U, C \cup D, V, f>$ be an IDIS in which $A_{1}, A_{2}, \cdots, A_{m} \subseteq C, A=\left\{A_{1}, A_{2}, \cdots, A_{m}\right\}, 0<\beta \leq 1$, then $\forall X \subseteq U$, the $V M G R S$ lower and upper approximations are denoted by $\underline{\sum_{i=1}^{m} A_{i} \beta}(X)$ and ${\overline{\sum_{i=1}^{m} A_{i}}}^{\beta}(X)$, respectively,

$$
\begin{aligned}
& \sum_{i=1}^{m} A_{i}(X)=\left\{x \in U: \forall A_{i} \in T, \operatorname{SIM}_{A_{i}}(x) \subseteq X\right\} . \\
& \overline{\sum_{i=1}^{m} A_{i}}(X)=\sim \sum_{\underline{i=1}}^{m} A_{i}(\sim X) .
\end{aligned}
$$

where $T \subseteq A$ and $\frac{|T|}{m} \geq \beta$.

Remark. By Definition 4, we can see that in $\sum_{i=1}^{m} A_{i}{ }^{\beta}(X)$, we need the number of the granulation $\operatorname{SIM}\left(A_{i}\right)$, which satisfy with the set containment to the target concept, to reach a certain amount. Such amount can be controlled by the threshold $\beta$.

By the lower and upper approximations $\sum_{i=1}^{m} A_{i}^{\beta}(X)$ and $\overline{\sum_{i=1}^{m} A_{i}} \beta(X)$, the VMGRS boundary region of $X$ is

$$
B N_{\sum_{i=1}^{m} A_{i}}^{\beta}(X)=\overline{\sum_{i=1}^{m} A_{i}}(X)-{\underline{\sum_{i=1}^{m} A_{i}}}^{\beta}(X) .
$$

Theorem 1.Let $I=<U, C \cup D, V, f>$ be an IDIS in which $A_{1}, A_{2}, \cdots, A_{m} \subseteq C, A=\left\{A_{1}, A_{2}, \cdots, A_{m}\right\}$, then $\forall X \subseteq U$, we have

$$
\begin{aligned}
& \text { 1. } \sum_{i=1}^{m} A_{i}{ }^{\frac{1}{m}}(X)=\sum_{i=1}^{m} A_{i}{ }^{O}(X), \\
& \overline{\sum_{i=1}^{m} A_{i}}{ }^{\frac{1}{m}}(X)=\overline{\sum_{i=1}^{m} A_{i}}{ }^{O}(X) \text {. } \\
& \text { 2. }{\underline{\sum_{i=1}^{m} A_{i}}}^{1}(X)=\underline{\sum_{i=1}^{m} A_{i}^{P}}(X) \text {, } \\
& {\overline{\sum_{i=1}^{m} A_{i}}}^{1}(X)={\overline{\sum_{i=1}^{m} A_{i}}}^{P}(X) \text {. }
\end{aligned}
$$

Proof. We only prove Eq. (1), the proof of Eq. (2) is similar to the proof of Eq. (1).

$\forall x \in U$, by Definition 2 and Definition 4, we have

$$
\begin{aligned}
x \in & \sum_{i=1}^{m} A_{i}^{\frac{1}{m}}(X) \\
& \Leftrightarrow \forall A_{i} \in T, \operatorname{SIM}_{A_{i}}(x) \subseteq X \text { where } T \subseteq A, \frac{|T|}{m} \geq \frac{1}{m} \\
& \Leftrightarrow \forall A_{i} \in T, \operatorname{SIM}_{A_{i}}(x) \subseteq X \text { where } T \subseteq A,|T| \geq 1 \\
& \Leftrightarrow \exists A_{i} \in A \text { s.t. } \operatorname{SIM}_{A_{i}}(x) \subseteq X \\
& \Leftrightarrow x \in \sum_{i=1}^{m} A_{i}(X)
\end{aligned}
$$


By Definition 4 and the above result, we have

$$
\begin{aligned}
{\overline{\sum_{i=1}^{m} A_{i}}}^{\frac{1}{m}}(X) & =\sim \sum^{\frac{m}{i=1} A_{i}^{\frac{1}{m}}}(\sim X) \\
& =\sim \sum_{i=1}^{m} A_{i}(\sim X) \\
& =\frac{\frac{}{m} O}{\sum_{i=1}^{m} A_{i}}(X)
\end{aligned}
$$

Remark. Through Theorem 1, we can see that if $\beta=\frac{1}{m}$, then the VMGRS lower approximation and upper approximation will degenerate to be Qian et al.'s OMGRS lower approximation and upper approximation in IDIS. On the other hand, if $\beta=1$, then the VMGRS lower approximation and upper approximation will degenerate to be Qian et al.'s PMGRS lower approximation and upper approximation in IDIS. From these point of views, the VMGRS is a generalization of both OMGRS and PMGRS.

Theorem 2.Let $I=<U, C \cup D, V, f>$ be an IDIS in which $A_{1}, A_{2}, \cdots, A_{m} \subseteq C$, suppose that $A=\left\{A_{1}, A_{2}, \cdots, A_{m}\right\}, 0<\beta \leq 1$, then $\forall X \subseteq U$, we have

$$
\begin{aligned}
& \text { 1. }{\overline{\sum_{i=1}^{m} A_{i}}}^{P}(X) \subseteq{\overline{\sum_{i=1}^{m} A_{i}}}^{\beta}(X) \subseteq{\overline{\sum_{i=1}^{m} A_{i}}}^{O}(X) . \\
& 2 . \underline{\sum_{i=1}^{m} A_{i}}{ }^{O}(X) \subseteq \underline{\sum_{i=1}^{m} A_{i}} \beta(X) \subseteq \underline{\sum_{i=1}^{m} A_{i}}{ }^{P}(X) .
\end{aligned}
$$

Proof. It can be derived directly from Definition 4 and Theorem 1.

Theorem 3.Let $I=<U, C \cup D, V, f>$ be an IDIS in which $A_{1}, A_{2}, \cdots, A_{m} \subseteq C$, suppose that $A=\left\{A_{1}, A_{2}, \cdots, A_{m}\right\}, \quad 0<\bar{\beta} \leq 1$, then $\forall X \subseteq U$, the following properties hold

$$
\begin{aligned}
& \text { 1. }{\underline{\sum_{i=1}^{m} A_{i}}}^{\beta}(X) \subseteq X \subseteq{\overline{\sum_{i=1}^{m} A_{i}}}^{\beta}(X) . \\
& \text { 2. } \sum_{i=1}^{m} A_{i} \beta(U)={\overline{\sum_{i=1}^{m} A_{i}}}^{\beta}(U)=U, \\
& \underline{\sum_{i=1}^{m} A_{i}}{ }^{\beta}(\emptyset)={\overline{\sum_{i=1}^{m} A_{i}}}^{\beta}(\emptyset)=\emptyset \\
& \text { 3. }{\overline{\sum_{i=1}^{m} A_{i}}}^{\beta}(\sim X)=\sim{\overline{\sum_{i=1}^{m} A_{i}}}^{\beta}(X) \text {, }
\end{aligned}
$$

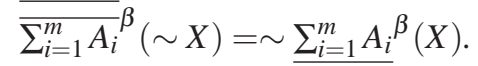

$$
\begin{aligned}
& \text { 4. } X \subseteq Y \Rightarrow \underline{\sum_{i=1}^{m} A_{i}{ }^{\beta}}(X) \subseteq \underline{\sum_{i=1}^{m} A_{i}^{\beta}}(Y) \text {, } \\
& {\overline{\sum_{i=1}^{m} A_{i}}}^{\beta}(X) \subseteq{\overline{\sum_{i=1}^{m} A_{i}}}^{\beta}(Y) . \\
& \text { 5. } \sum_{i=1}^{m} A_{i}{ }^{\beta}\left(\sum_{i=1}^{m} A_{i}{ }^{\beta}(X)\right)=\sum_{i=1}^{m} A_{i}{ }^{\beta}(X),
\end{aligned}
$$

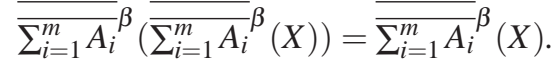

$$
\begin{aligned}
& \text { 6. } \beta_{1} \leq \beta_{2} \Rightarrow \underline{\sum_{i=1}^{m} A_{i}^{\beta_{1}}}(X) \supseteq \underline{\sum_{i=1}^{m} A_{i}^{\beta_{2}}}(X), \\
& {\overline{\sum_{i=1}^{m} A_{i}}}^{\beta_{1}}(X) \subseteq{\overline{\sum_{i=1}^{m} A_{i}}}^{\beta_{2}}(X) .
\end{aligned}
$$

Proof.
1. (a)Let $x \in \sum_{i=1}^{m} A_{i}{ }^{\beta}(X), \forall A_{i} \in T$, there must be $\operatorname{SIM}_{A_{i}}(x) \subseteq X$, where $T \subseteq A$ and $|T| / m \geq \beta$. Since $x \in \operatorname{SIM}_{A_{i}}(x)$, then $x \in X$. So it follows that $\sum_{i=1}^{m} A_{i}^{\beta}(X) \subseteq X$.

(b)Let $x \notin{\overline{\sum_{i=1}^{m} A_{i}}}^{\beta}(X)$, then $x \in \sum_{i=1}^{m} A_{i}^{\beta}(\sim X)$, for $\forall A_{i} \in T$, there must be $\operatorname{SIM}_{A_{i}}(x) \subseteq(\sim X)$, then $x \in$ $(\sim X)$, hence $x \notin X$. Thus, $X \subseteq{\overline{\sum_{i=1}^{m} A_{i}}}^{\beta}(X)$.

2. (a)From 1, one can get that $\sum_{i=1}^{m} A_{i}^{\beta}(U) \subseteq U$, And if $x \in U$, then, for each $\overline{A_{i} \in T}$, there must be $\operatorname{SIM}_{A_{i}}(x) \subseteq U$,where $T \subseteq A$ and $|T| / m \geq \beta$. hence, $x \in \underline{\sum_{i=1}^{m} A_{i}^{\beta}}(U)$ and $U \in \underline{\sum_{i=1}^{m} A_{i}^{\beta}}(U)$. Thus, $\sum_{i=1}^{m} A_{i} \bar{\beta}(U)=U$.

(b)From 1, we know that $U \subseteq{\overline{\sum_{i=1}^{m} A_{i}}}^{\beta}(U)$, and $\bar{\sum}_{i=1}^{m} A_{i}^{\beta}(U) \subseteq U$ hold clearly. Thus, ${\overline{\sum_{i=1}^{m} A_{i}}}^{\beta}(U)=U$

(c)From 1, we know that $\sum_{i=1}^{m} A_{i}{ }^{\beta}(\emptyset) \subseteq \emptyset$. And $\emptyset \subseteq$ $\sum_{i=1}^{m} A_{i}^{\beta}(\emptyset)$ hold clearly. Thus, $\sum_{i=1}^{m} A_{i}^{\beta}(\emptyset)=\emptyset$.

(d)From Definition 4, we know that $\overline{\sum_{i=1}^{m} A_{i}} \beta(\emptyset)=\sim$ $\sum_{i=1}^{m} A_{i}{ }^{\beta}(U)$. From 2.(b), we know $\sum_{i=1}^{m} A_{i}{ }^{\beta}(U)=$ $U$. Thus, ${\overline{\sum_{i=1}^{m} A_{i}}}^{\beta}(\emptyset)=\emptyset$

3.From Definition 3, we know that $\sum_{i=1}^{m} A_{i}{ }^{\beta}(\sim X)=\sim$

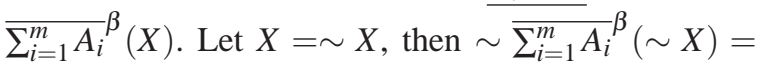
$\sum_{i=1}^{m} A_{i}^{\beta}(X)$.

4. (a)Let $x \in \sum_{i=1}^{m} A_{i}^{\beta}(X), \forall A_{i} \in T$, there must be $\operatorname{SIM}_{A_{i}}(x) \subseteq X$, where $T \subseteq A$ and $|T| / m \geq \beta$. since $X \subseteq Y$, then $\operatorname{SIM}_{A_{i}}(x) \subseteq Y$, hence $\underline{\sum_{i=1}^{m} A_{i}^{\beta}}(X) \subseteq \underline{\sum_{i=1}^{m} A_{i}^{\beta}}(Y)$.

(b) Since $X \subseteq Y$, then $\sim Y \subseteq \sim X$ and $\sum_{i=1}^{m} A_{i}{ }^{\beta}(\sim \bar{Y}) \subseteq \sum_{i=1}^{m} A_{i}{ }^{\beta}(\sim X)$, from (3), we know that $\overline{\sum_{i=1}^{m} A_{i}} \beta \overline{(X) \subseteq \bar{\sum}_{i=1}^{m} A_{i}} \beta(Y)$.

5. (a)From 1 , we easily know that $\sum_{i=1}^{m} A_{i}{ }^{\beta}\left(\sum_{i=1}^{m} A_{i}^{\beta}(X)\right) \subseteq \sum_{i=1}^{m} A_{i}^{\beta}(X)$. Suppose $\overline{x \in \sum_{i=1}^{m}} \overline{A_{i} \beta(X)}$, then for each $A_{i} \in T$, there must be $\overline{S I M_{A_{i}}(x)} \subseteq X$, where $T \subseteq A$ and $|T| / m \geq \beta$, hence $\sum_{i=1}^{m} A_{i}^{\beta}\left(\operatorname{SIM}_{A_{i}}(x)\right) \subseteq \sum_{i=1}^{m} A_{i}{ }^{\beta}(X)$. But $\left.\sum_{i=1}^{m} A_{i} \overline{\operatorname{SIM}_{A_{i}}}(x)\right)=\operatorname{SIM}_{A_{i}}(x)$, then we can have that $\operatorname{SIM}_{A_{i}}(x) \subseteq \sum_{i=1}^{m} A_{i}^{\beta}(X), \quad$ Hence $x \in \underline{\sum_{i=1}^{m} A_{i}{ }^{\beta}}\left(\underline{\sum_{i=1}^{m} A_{i}{ }^{\beta}}(X) \overline{A^{\beta}(X)}\right.$. Then, we get that,

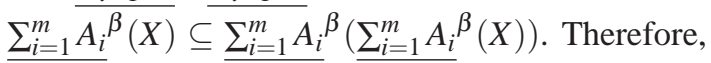
$\overline{\sum_{i=1}^{m} A_{i}}{ }^{\beta}(X)=\overline{\sum_{i=1}^{m} A_{i}}{ }^{\beta}\left(\overline{\sum_{i=1}^{m} A_{i}}{ }^{\beta}(X)\right)$.

(b) $\overline{\text { Form }} 3$ and 5(a), we know that ${\overline{\sum_{i=1}^{m} A_{i}}}^{\beta}\left({\overline{\sum_{i=1}^{m} A_{i}}}^{\beta}(X)\right)={\overline{\sum_{i=1}^{m} A_{i}}}^{\beta}\left(\sim \sum_{i=1}^{m} A_{i}{ }^{\beta}(\sim\right.$ $X))=\sim{\underline{\sum_{i=1}^{m} A_{i}}}^{\beta}\left(\underline{\sum_{i=1}^{m} A_{i} \beta}(\sim X)\right)=\sim \overline{\sum_{i=1}^{m} A_{i}}{ }^{\beta}(\sim$ $\left.X))={\overline{\sum_{i=1}^{m} A_{i}}}^{\beta}(X)\right)$. 
6.Let $x \in \sum_{i=1}^{m} A_{i}{ }^{\beta_{2}}(X)$, for each $A_{i} \in T$, there must be $\operatorname{SIM}_{A_{i}}(x) \subseteq X$, where $T \subseteq A$ and $|T| / m \geq \beta_{2}$. Since $\beta_{1} \leq \beta_{2}$, then $|T| / m \geq \beta_{1}$, hence $x \in \sum_{i=1}^{m} A_{i}{ }^{\beta_{1}}(X)$. Similarity, it is a trivial to prove ${\overline{\sum_{i=1}^{m} A_{i}}}^{\beta_{1}}(X) \subseteq{\overline{\sum_{i=1}^{m} A_{i}}}^{\beta_{2}}(X)$

This completes the proof.

Theorem 3 shows the basic properties about the VMGRS in IDIS. 1 says that the VMGRS lower approximation is included into the target concept and the VMGRS upper approximation includes the target concept. 2 shows the normality of the VMGRS. 3 expresses the complement properties of the VMGRS. 4 says the monotonic properties about the VMGRS in terms of the monotonic varieties of the target concepts. 5 says the idempotents of the VMGR. 6 says the monotonic properties about the VMGRS in terms of the monotonic varieties of the threshold.

Example 1.We then use an example to illustrate the VMGRS in incomplete decision information system. Table 1 depicts an incomplete decision information system about the emporium investment project. "Locus", "Investment", and "Population density" are the conditional attributes of the system, and "Decision" is the decision attribute (in the sequel, $a_{1}, a_{2}, a_{3}$, and $d$ will stand for "Locus", "Investment", "Population density", and "Decision", respectively).

Let $A=\left\{A_{1}, A_{2}, A_{3}\right\}=\left\{\left\{a_{1}, a_{2}\right\},\left\{a_{2}, a_{3}\right\},\left\{a_{1}, a_{3}\right\}\right\}$, since the decision attribute determines a partition on the universe such that $U /\{d\}=\left\{D_{1}, D_{2}\right\}=$ $\left\{\left\{x_{3}, x_{4}, x_{5}, x_{7}, x_{9}\right\},\left\{x_{1}, x_{2}, x_{6}, x_{8}, x_{10}\right\}\right\}$, then by Definition 2, we have

$$
\begin{aligned}
& \frac{\sum_{i=1}^{3} A_{i}{ }^{O}}{}\left(D_{1}\right)=\left\{x_{3}, x_{5}, x_{7}, x_{9}\right\} . \\
& \overline{\sum_{i=1}^{3} A_{i}}{ }^{O}\left(D_{2}\right)=\left\{x_{1}, x_{2}, x_{8}, x_{10}\right\} . \\
& \overline{\sum_{i=1}^{3} A_{i}} O\left(D_{1}\right)=\left\{x_{3}, x_{4}, x_{5}, x_{6}, x_{7}, x_{9}\right\} . \\
& \overline{\sum_{i=1}^{3} A_{i}} O\left(D_{2}\right)=\left\{x_{1}, x_{2}, x_{4}, x_{6}, x_{8}, x_{10}\right\} .
\end{aligned}
$$

By Definition 3, we have

$$
\begin{aligned}
& \frac{\sum_{i=1}^{3} A_{i}^{P}}{P}\left(D_{1}\right)=\emptyset . \\
& \frac{\sum_{i=1}^{3} A_{i}^{P}}{\sum^{3}}{ }^{P}\left(D_{2}\right)=\left\{x_{10}\right\} . \\
& \frac{\sum_{i=1}^{3} A_{i}}{\sum_{i=1}^{3} A_{i}} P\left(D_{1}\right)=\left\{x_{1}, x_{2}, x_{3}, x_{4}, x_{5}, x_{6}, x_{7}, x_{8}, x_{9}\right\} . \\
& \left.x_{1}, x_{2}, x_{3}, x_{4}, x_{5}, x_{6}, x_{7}, x_{8}, x_{9}, x_{10}\right\} .
\end{aligned}
$$

Suppose $\beta=0.5$, then by Definition 4 , we have

$$
\begin{aligned}
& \sum_{i=1}^{3} A_{i}^{\beta}\left(D_{1}\right)=\left\{x_{3}, x_{5}, x_{9}\right\} . \\
& \underline{\underline{\sum_{i=1}^{3} A_{i}^{\beta}}}{ }^{\beta}\left(D_{2}\right)=\left\{x_{1}, x_{8}, x_{10}\right\} \text {. } \\
& \overline{\sum_{i=1}^{3} A_{i}}{ }^{\beta}\left(D_{1}\right)=\left\{x_{2}, x_{3}, x_{4}, x_{5}, x_{6}, x_{7}, x_{9}\right\} . \\
& {\overline{\sum_{i=1}^{3} A_{i}}}^{\beta}\left(D_{2}\right)=\left\{x_{1}, x_{2}, x_{4}, x_{6}, x_{7}, x_{8}, x_{10}\right\} \text {. }
\end{aligned}
$$

Through the above results, we have

$$
\begin{aligned}
& \frac{\sum_{i=1}^{3} A_{i}^{P}}{{ }^{P}}\left(D_{1}\right) \subseteq \underline{\sum_{i=1}^{3} A_{i}^{\beta}}\left(D_{1}\right) \subseteq \underline{\sum_{i=1}^{3} A_{i}{ }^{O}}\left(D_{1}\right) . \\
& \underline{\sum_{i=1}^{3} A_{i}}{ }^{P}\left(D_{2}\right) \subseteq \underline{\sum_{i=1}^{3} A_{i}{ }^{\beta}}\left(D_{2}\right) \subseteq \underline{\sum_{i=1}^{3} A_{i}}{ }^{O}\left(D_{2}\right) .
\end{aligned}
$$

Table 1: An incomplete decision information table of the emporium investment project

\begin{tabular}{ccccc}
\hline Project & Locus & Investment & $\begin{array}{c}\text { Population } \\
\text { density }\end{array}$ & Decision \\
\hline$x_{1}$ & Common & High & High & Yes \\
$x_{2}$ & Bad & High & High & Yes \\
$x_{3}$ & Bad & $*$ & Small & No \\
$x_{4}$ & Bad & Low & $*$ & No \\
$x_{5}$ & Bad & Low & Small & No \\
$x_{6}$ & Bad & $*$ & Medium & Yes \\
$x_{7}$ & Common & High & Medium & No \\
$x_{8}$ & Good & $*$ & Medium & Yes \\
$x_{9}$ & Bad & Low & Bad & Yes \\
$x_{10}$ & Good & High & High & Yes \\
\hline
\end{tabular}

$$
\begin{aligned}
& {\overline{\sum_{i=1}^{3} A_{i}}}^{O}\left(D_{1}\right) \subseteq{\overline{\sum_{i=1}^{3} A_{i}}}^{\beta}\left(D_{1}\right) \subseteq{\overline{\sum_{i=1}^{3} A_{i}}}^{P}\left(D_{1}\right) . \\
& {\overline{\sum_{i=1}^{3} A_{i}}}^{O}\left(D_{2}\right) \subseteq{\overline{\sum_{i=1}^{3} A_{i}}}^{\beta}\left(D_{2}\right) \subseteq{\overline{\sum_{i=1}^{3} A_{i}}}^{P}\left(D_{2}\right) .
\end{aligned}
$$

these results show the correctness of Theorem 2 .

\section{Measurements}

Uncertainty of a set (category) is due to the existence of a borderline region. The greater the borderline region of a set, the lower is the accuracy of the set. To more precisely express this idea, we introduce some accuracy measurements as following.

Definition 5.Let $I=<U, C \cup D, V, f>$ be an IDIS in which $A_{1}, A_{2}, \cdots, A_{m} \subseteq C, \quad A=\left\{A_{1}, A_{2}, \cdots, A_{m}\right\}$, $0<\beta \leq 1$, then $\forall X \subseteq U(X \neq \emptyset)$. The accuracies degrees of $X$ in terms of OMGRS, PMGRS and VMGRS in incomplete decision information system are defined respectively as

$$
\begin{aligned}
& \alpha_{O}\left(\sum_{i=1}^{m} A_{i}, X\right)=\frac{\left|\underline{\sum i=1}_{i=1}^{m} A_{i}^{O}(X)\right|}{\left|{\overline{\sum_{i=1}^{m} A_{i}}}^{O}(X)\right|} \\
& \alpha_{P}\left(\sum_{i=1}^{m} A_{i}, X\right)=\frac{\left|\sum_{i=1}^{m} A_{i}^{P}(X)\right|}{\left|{\overline{\sum_{i=1}^{m} A_{i}}}^{P}(X)\right|} \\
& \alpha_{\beta}\left(\sum_{i=1}^{m} A_{i}, X\right)=\frac{\left|\underline{\sum_{i=1}^{m} A_{i}^{\beta}}(X)\right|}{\left|\overline{\sum_{i=1}^{m} A_{i}}{ }^{\beta}(X)\right|}
\end{aligned}
$$

Definition 6.Let $I=<U, C \cup D, V, f>$ be an IDIS in which $A_{1}, A_{2}, \cdots, A_{m} \subseteq C, \quad A=\left\{A_{1}, A_{2}, \cdots, A_{m}\right\}$, $0<\beta \leq 1, U / D=\left\{D_{1}, D_{2}, \ldots D_{r}\right\}$ be the partition induced by the decision attribute sets $D$. The qualities of approximations of $D$ by $A$, also called the degrees of dependencies in terms of the OMGRS, PMGRS and VMGRS in incomplete decision information system are 
defined respectively as

$$
\begin{aligned}
& \gamma_{O}\left(\sum_{i=1}^{m} A_{i}, D\right)=\frac{\sum\left\{\left|\sum_{i=1}^{m} A_{i}^{O}\left(D_{r}\right)\right|: D_{r} \in D\right\}}{|U|} \\
& \gamma_{P}\left(\sum_{i=1}^{m} A_{i}, D\right)=\frac{\sum\left\{\left|\sum_{i=1}^{m} A_{i}^{P}\left(D_{r}\right)\right|: D_{r} \in D\right\}}{|U|} \\
& \gamma_{\beta}\left(\sum_{i=1}^{m} A_{i}, D\right)=\frac{\sum\left\{\left|\sum_{i=1}^{m} A_{i}^{\beta}\left(D_{r}\right)\right|: D_{r} \in D\right\}}{|U|}
\end{aligned}
$$

Definition 7.Let $I=<U, C \cup D, V, f>$ be an IDIS in which $A_{1}, A_{2}, \cdots, A_{m} \subseteq C, \quad A=\left\{A_{1}, A_{2}, \cdots, A_{m}\right\}$, $0<\beta \leq 1$, then $\forall X \subseteq U(X \neq \emptyset)$, the approximated degrees of $X$ in terms of OMGRS, PMGRS and VMGRS in incomplete decision information system are defined respectively as

$$
\begin{aligned}
& \pi_{O}\left(\sum_{i=1}^{m} A_{i}, X\right)=\frac{\left|\sum_{i=1}^{m} A_{i}{ }^{O}(X)\right|}{|X|} \\
& \pi_{P}\left(\sum_{i=1}^{m} A_{i}, X\right)=\frac{\left|\sum_{i=1}^{m} A_{i}{ }^{P}(X)\right|}{|X|} \\
& \pi_{\beta}\left(\sum_{i=1}^{m} A_{i}, X\right)=\frac{\left|\sum_{i=1}^{m} A_{i}^{\beta}(X)\right|}{|X|}
\end{aligned}
$$

Theorem 4.Let $I=<U, C \cup D, V, f>$ be an IDIS in which $A_{1}, A_{2}, \cdots, A_{m} \subseteq C, \quad A=\left\{A_{1}, A_{2}, \cdots, A_{m}\right\}$, $0<\beta \leq 1$, then $\forall X \subseteq U(X \neq \emptyset)$. The accuracies degrees and the approximated degrees of $X$ have the following properties:

$$
\begin{aligned}
& \alpha_{P}\left(\sum_{i=1}^{m} A_{i}, X\right) \leq \alpha_{\beta}\left(\sum_{i=1}^{m} A_{i}, X\right) \leq \alpha_{O}\left(\sum_{i=1}^{m} A_{i}, X\right) \\
& \pi_{P}\left(\sum_{i=1}^{m} A_{i}, X\right) \leq \pi_{\beta}\left(\sum_{i=1}^{m} A_{i}, X\right) \leq \pi_{O}\left(\sum_{i=1}^{m} A_{i}, X\right)
\end{aligned}
$$

Proof. From Theorem 2, we know that ${\overline{\sum_{i=1}^{m} A_{i}}}^{P}(X) \subseteq{\overline{\sum_{i=1}^{m} A_{i}}}^{\beta}(X) \subseteq{\overline{\sum_{i=1}^{m} A_{i}}}^{O}(X) \quad$ and $\sum_{i=1}^{m} A_{i}{ }^{O}(X) \subseteq \sum_{i=1}^{m} A_{i}{ }^{\beta}(X) \subseteq \sum_{i=1}^{m} A_{i}{ }^{P}(X)$. then

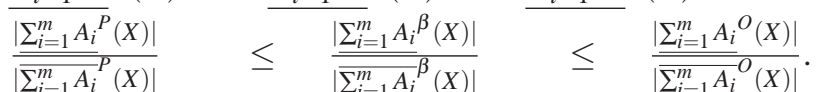
thus, $\alpha_{P}\left(\sum_{i=1}^{m} A_{i}, X\right) \leq \alpha_{\beta}\left(\sum_{i=1}^{m} A_{i}, X\right) \leq \alpha_{O}\left(\sum_{i=1}^{m} A_{i}, X\right)$. Similarity, it is a trivial to prove $\pi_{P}\left(\sum_{i=1}^{m} A_{i}, X\right) \leq \pi_{\beta}\left(\sum_{i=1}^{m} A_{i}, X\right) \leq \pi_{O}\left(\sum_{i=1}^{m} A_{i}, X\right)$.

Theorem 5.Let $I=<U, C \cup D, V, f>$ be an IDIS in which $A_{1}, A_{2}, \cdots, A_{m} \subseteq C, \quad A=\left\{A_{1}, A_{2}, \cdots, A_{m}\right\}$, $0<\beta \leq 1, U / D=\left\{D_{1}, D_{2}, \ldots D_{r}\right\}$ be the partition induced by the decision attribute sets $D$. the dependencies degrees of $D$ have the following properties:

$\gamma_{\beta}\left(\sum_{i=1}^{m} A_{i}, D\right) \leq \gamma_{\beta}\left(\sum_{i=1}^{m} A_{i}, D\right) \leq \gamma_{O}\left(\sum_{i=1}^{m} A_{i}, D\right)$

Proof. It can be derived directly from Theorem 2 and Definition 6.
Table 2: Data sets descrition

\begin{tabular}{ccccc}
\hline Date sets & Samples & Data type & $\begin{array}{c}\text { Conditional } \\
\text { density }\end{array}$ & $\begin{array}{c}\text { Decision } \\
\text { calss }\end{array}$ \\
\hline $\begin{array}{c}\text { Breast-cancer } \\
\text {-wisconsin } \\
\text { Agaricus } \\
\text {-lepiota }\end{array}$ & 699 & Incomplete & 9 & 2 \\
\hline
\end{tabular}

Table 3: Comparisons of lower approximation in breast-cancerwisconsin data set

\begin{tabular}{ccc}
\hline Lower & \multicolumn{2}{c}{ Descision classes } \\
\cline { 2 - 3 } Approximations & $D_{1}$ & $D_{2}$ \\
\hline OMGRS & 434 & 239 \\
VMGRS $(\beta=0.2)$ & $\mathbf{4 3 4}$ & $\mathbf{2 3 9}$ \\
VMGRS $(\beta=0.5)$ & $\mathbf{2 3 1}$ & $\mathbf{2 2 8}$ \\
VMGRS $(\beta=1)$ & $\mathbf{4 3}$ & $\mathbf{1 6 3}$ \\
PMGRS & 43 & 163 \\
\hline
\end{tabular}

\section{Experimental Analysis}

In the following, through experimental analysis, we will illustrate the relations among VMGRS, OMGRS and PMGRS in IDIS. We have downloaded two public data sets from UCI Repository of machine learning databases, which are described in Table 2.

Here, we compare the lower and upper Approximations in our VMGRS with that in OMGRS and PMGRS on these two practical data sets. The comparisons of the lower and upper approximations in Breast-cancer-wisconsin data set are shown in Table 3 and Table 4.

Table 3 and Table 4 show the number of elements of lower and upper approximations in terms of three different MGRS approaches. We can see that if $\beta=0.2$, then the lower and upper approximations in VMGRS are same to that in OMGRS; if $\beta=1$, then the lower and upper approximations in VMGRS are same to that in PMGRS. Such results are consistent to Theorem 1.

In particular, if $\beta=0.5$, then the Lower and upper approximations in VMGRS are between that in OMGRS and PMGRS. Such results are consistent to Theorem 2.

Similarity, it is not difficult to draw the same conclusions from Table 5 and Table 6.

So, we can conclude that VMGRS is a generalization of both OMGRS and PMGRS, OMGRS and PMGRS are two type especial instances of VMGRS

\section{Conclusions}

In this paper, the VMGRS is proposed. In our VMGRS approach, a threshold is used to control the number of granulations, which satisfy with the inclusion condition 
Table 4: Comparisons of upper approximation in breast-cancerwisconsin data set

\begin{tabular}{ccc}
\hline Upper & \multicolumn{2}{c}{ Descision classes } \\
\cline { 2 - 3 } Approximations & $D_{1}$ & $D_{2}$ \\
\hline OMGRS & 460 & 265 \\
VMGRS $(\beta=0.2)$ & $\mathbf{4 6 0}$ & $\mathbf{2 6 5}$ \\
VMGRS $(\beta=0.5)$ & $\mathbf{4 7 1}$ & $\mathbf{4 6 8}$ \\
VMGRS $(\beta=1)$ & $\mathbf{5 3 6}$ & $\mathbf{6 5 6}$ \\
PMGRS & 536 & 656 \\
\hline
\end{tabular}

Table 5: Comparisons of lower approximation in agaricuslepiota data set

\begin{tabular}{ccc}
\hline Lower & \multicolumn{2}{c}{ Descision classes } \\
\cline { 2 - 3 } Approximations & $D_{1}$ & $D_{2}$ \\
\hline OMGRS & 4196 & 3916 \\
VMGRS $(\beta=0.2)$ & $\mathbf{4 1 9 6}$ & $\mathbf{3 9 1 6}$ \\
VMGRS $(\beta=0.5)$ & $\mathbf{3 9 5 6}$ & $\mathbf{3 7 5 6}$ \\
VMGRS $(\beta=1)$ & $\mathbf{1 2 2 8}$ & $\mathbf{2 1 6 0}$ \\
PMGRS & 1228 & 2160 \\
\hline
\end{tabular}

Table 6: Comparisons of upper approximation in agaricuslepiota data set

\begin{tabular}{ccc}
\hline Upper & \multicolumn{2}{c}{ Descision classes } \\
\cline { 2 - 3 } Approximations & $D_{1}$ & $D_{2}$ \\
\hline OMGRS & 4208 & 3928 \\
VMGRS $(\beta=0.2)$ & $\mathbf{4 2 0 8}$ & $\mathbf{3 9 2 8}$ \\
VMGRS $(\beta=0.5)$ & $\mathbf{4 3 6 8}$ & $\mathbf{4 1 6 8}$ \\
VMGRS $(\beta=1)$ & $\mathbf{5 9 6 4}$ & $\mathbf{6 8 9 6}$ \\
PMGRS & 5964 & 6896 \\
\hline
\end{tabular}

between the knowledge granules and the target. Not only the theoretical discussions, but also the experimental analyses show that our VMGRS is a generalization of the OMGRS and PMGRS in IDIS. In our further researching, the reduction in terms of the proposed incomplete VMGRS is an interesting topic to be addressed.

\section{Acknowledgement}

The first author acknowledges the financial support by the Natural Science Foundation of China (No. 61100116), Natural Science Foundation of Jiangsu Province of China (No. BK2011492), Natural Science Foundation of Jiangsu Higher Education Institutions of China (No. 11KJB520004).

The authors are grateful to the anonymous referee for a careful checking of the details and for helpful comments that improved this paper.

\section{References}

[1] Z. Pawlak, Rough sets-theoretical aspects of reasoning about data, Kluwer Academic Publishers, (1992).

[2] Z. Pawlak, A. Skowron, Rough sets: Some extensions, Information Sciences, 177, 28-40 (2007).

[3] J. Baszczyski, S. Greco, R. Slowinski, Multi-criteria classification - a new scheme for application of dominancebased decision rules, European Journal of Operational Research, 181, 1030-1044 (2007).

[4] H. M. Abu-Donia, Multi knowledge based rough approximations and applications, Knowledge- Based Systems, 26, 20-29 (2012).

[5] X. B. Yang, J. Xie, X. N. Song, et al, Credible rules in incomplete decision system based on descriptors, Knowledge-Based-Systems, 22, 8-17 (2009).

[6] Y. Y. Yao, Three-way decisions with probabilistic rough sets, Information Sciences, 180, 341-353 (2010).

[7] M. A. Khan, M. Banerjee, Formal reasoning with rough sets in multiple-souce approximation systems, International Journal of Approximate Reasoning, 49, 466-477 (2008).

[8] T. Y. Lin, Introduction to special issues on data mining and granular computing, International Journal of Approximate Reasoning, 40, 1-2 (2005).

[9] N. M. Parthalain, Q. Shen, Exploring the boundary region of tolerance rough sets for feature selection, Pattern Recognition, 42, 655-667 (2009).

[10] Y. H. Qian, J. Y. Liang, W. Pedrycz, C. Y. Dang, Positive approximation: an accelerator for attribute reduction in rough set theory, Artificial Intelligence, 174, 597-618 (2010).

[11] S. Tsumoto, Automated discovery of positive and negative knowledge in clinical databases, IEEE Engineering in Medicine and Biology Society, 19, 56-62 (2000).

[12] R. Slowinski, D. Vanderpooten, A generalized definition of rough approximations based on similarity, IEEE Transaction on Knowledge and Data Engineering, 12, 331-336 (2000).

[13] M. Kryszkiewicz, Rough sets approach to incomplete information systems, Information Sciences 112, 39-49 (1998).

[14] D. Kim, Data classification based on tolerant rough set, Pattern Recognition, 34, 1613-1624 (2001).

[15] W. H. Xu, Q. R. Wang, X.T. Zhang, Multi-Granulation Fuzzy Rough Sets in a Fuzzy Tolerance Approximation Space, International Journal of Fuzzy Systems, 13, 246-259 (2011).

[16] S. Greco, B. Matarazzo, Slowinski, Rough approximation by dominance relations, International Journal of Intelligent Systems, 17, 153-171 (2002).

[17] W. Zhu, F. Y. Wang, On three types of covering-based rough sets, IEEE Transactons on Knowledge and Engineering, 19, 1131-1144 (2007).

[18] G. L. Liu, Y. Sai, A comparison of two types of rough sets induced by coverings, International Journal of Approximate Reasoning, 50, 521-528 (2009).

[19] D. Dubios, H. Prade, Rough fuzzy sets and fuzzy rough sets, International Journal of General Systems, 17, 191-209 (1990).

[20] Q. H. Hu, D. R. Yu, Z. X. Xie, J. F. Liu, Fuzzy probabilistic approximation spaces and their information measures, IEEE Transations on Fuzzy System, 14, 191-206 (2006). 
[21] Q. H. Hu, L. Zhang, D. G. Chen, W. Pedrycz, D. R. Yu, Gaussian kernel based fuzzy rough sets: Model, uncertainty measures and applications. International Journal of Approximate Reasoning, 51, 453-471 (2010).

[22] Y. Ouyang, Z. D. Wang, H. P. Zhang, On fuzzy rough sets based on tolerance relations, Information Sciences, 180, 532542 (2010).

[23] W. Ziarko, Variable precision rough set model, Journal of Computer and System Science, 46, 39-59 (1993).

[24] Y. Y. Yao, Information granulation and rough approximation, International Journal of Intelligent Systems, 16, 87-104 (2001).

[25] Y. H. Qian, J. Y. Liang, C. Y. Dang. Incomplete multigranulation rough set, IEEE Transactions on Systems, Man and Cybernetics, Part A, 20, 420-431 (2010).

[26] W. H. Xu, W. X. Sun, X. Y. Zhang, W. X. Zhang, Multiple granulation rough set approach to ordered information systems, International Journal of General Systemsl, 41, 475501 (2012).

[27] Y. H. Qian, J. Y. Liang, Y. Y. Yao, C. Y. Dang. MGRS: a multi-granulation rough set, Information Sciences, 180, 949970 (2010).

[28] Y. H. Qian, J. Y. Liang, W. Wei, Pessimistic rough decision, In: Second International Worshop on Rough Sets Theory, 440-449, (2010).

[29] G. P. Lin, Y. H. Qian, J. J. Li, NMGRS: Neighborhoodbased multigranulation rough sets, International Journal of Approximate Reasoning, 53, 1080-1093 (2012).

[30] X. B. Yang, X. N. Song, H. L. Dou, J. Y. Yang, Multigranulation rough set: from crisp to fuzzy case, Annals of Fuzzy Mathematics and Informatics, 1, 55-70 (2011).

[31] W. H. Xu, W. X. Sun, X. Y. Zhang, W. X. Zhang, Multiple granulation rough set approach to ordered information systems, International Journal of General Systems, 41, 475501 (2012).

[32] Y. H. She, X. L. He, On the structure of the multigranulation rough set model , Knowledge-Based Systems, 32, 81-92 (2012).

[33] M. Zhang, Z. M. Tang, W. Y. Xu, X. B. Yang, A Variable Muitlgranulation Rough Sets Approach, Lecture Notes in Lecture Notes in Computer Science, 6840, 315-322 (2012).

[34] W. Z. Wu, Y. Leung. Theory and applications of granular labelled partitions in multi-scale decision tables, Information Sciences, 181, 3878-3897(2011).

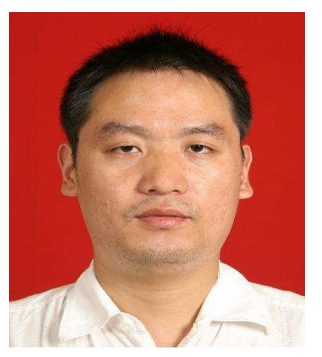

Ming Zhang is currently a $\mathrm{PhD}$ candidate in the School of computer science and technology, Nanjing University of science and technology. He received his BS degree and MS degree in Computer science from Jiangsu University of Science and Technology in 2002 and 2005 respectively. His research interests include rough sets, granular computing and intelligence information processing.

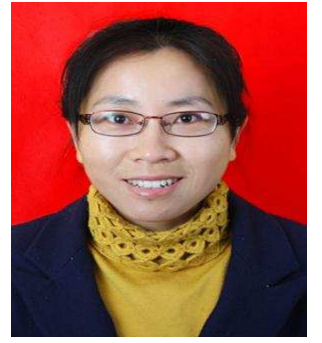

Weiyan $\mathrm{Xu}$ is currently a lecturer in the college of Mathematics and Physics, Jiangsu University of Science and Technology. She received her B.S. degree in Mathematics science and M.S. degree in system analysis and integration from Nanjing Normal University, in 2002 and 2005 respectively. Her research interests include Rough Sets and Fuzzy sets.

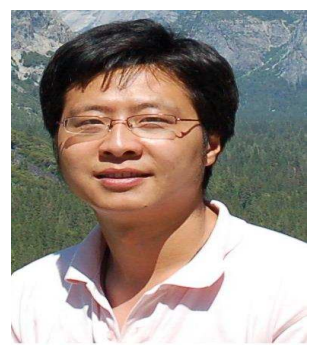

Xibei Yang is currently a lecturer in Computer Science and Engineering, Jiangsu University of Science and Technology. He received his $\mathrm{PhD}$ degree in Computer science from Nanjing University of science and technology in 2009, and MS degree in Computer science from Jiangsu University of Science and Technology in 2006. His research interests include Rough Sets, granular computing and intelligence information processing.

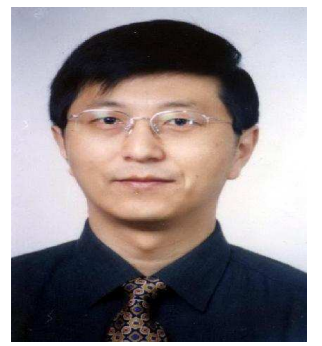

Zhenmin Tang is
currently a professor
in School of computer science and technology, Nanjing University of science and technology. He received his MS degree in Computer science and $\mathrm{PhD}$ degree in pattern recognition from Nanjing University of science and technology in 1988 and 2002 respectively. He research interests include pattern recognition and intelligence information processing. 